\title{
Haiti, four months after the earthquake
}

Published at www.cmaj.ca on June 1

$\mathrm{F}$ our months after the earthquake, the destruction and devastation in Port au Prince is overwhelming and of epic proportions. The general appearance of the city is like that of Gaza after an air raid, but worse.

The streets are strewn with rubble and shattered homes, teeming with pedestrians trying to dodge mopeds, cars and buses painted in gaudy bright colours. Everywhere one looks are makeshift tents constructed of tarps and plastic bags packed tightly together, collectively housing more than one million displaced persons. Many others remain in buildings that should already have been torn down, if only there were sufficient resources to do so, as they pose a continued risk to the safety of those who continue to inhabit or even come close to them.

The team I was with came from Children's Hospital in Boston, Massachusetts, and stayed for nine days at the State University Hospital of Haiti, where we worked with members of the Department of Pediatrics. Electricity and running water were intermittent; the conditions for patients bore no comparison to those most of us would consider the barest of minimum back home. There were no sheets or bedclothes, no food provided for the patients or their families, and medications and IV fluids needed to be purchased by the families at pharmacies or pushcarts outside the hospital.

Our tasks were quite varied, and ranged from attending kids in the emergency room and the pediatric intensive care tent to running transports or helping out with anesthesia. We spent a lot of time working with, and giving formal and informal teaching to the residents and to the Haitian attending physicians, who are among the most dedicated and impressive people I have

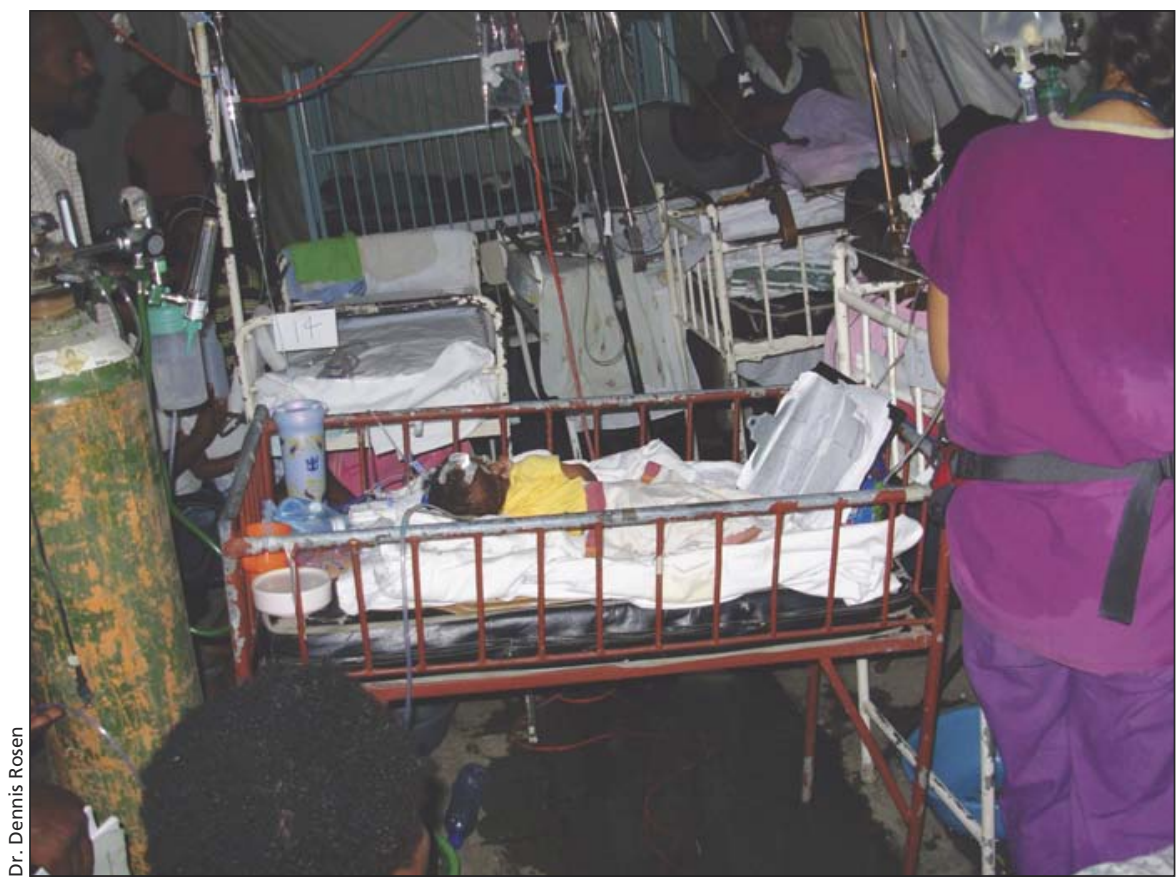

The conditions for patients in Haiti bear no resemblance to those in North American hospitals, says Boston, Massachusetts, physician Dr. Dennis Rosen.

ever met. Despite not having been paid for more than nine months, these physicians (and nurses) continued to show up to work each day in clean, pressed shirts, delivering care with thoroughness and compassion that was tremendously inspiring.

There were four pediatric tents at the university hospital, each about 30 feet by 10 feet. The intensive care tent held, at one point, 28 infants and children in rusty cribs and on rickety cots, often doubled and tripled up.

Each pediatric tent had one to two caregivers. In one tent, a child who had been abandoned was being cared for by other parents. The tent flaps were kept down at night to prevent malaria, though this was not a great strategy to prevent the spread of the tuberculosis, which several of the children and their parents had been diagnosed with.

When there was electricity, the fans worked, though it remained above 100 degrees Fahrenheit; when not, it was hard to be inside the tents without becoming drenched with sweat after a few minutes.

Supplies were erratic and often impossible to come by. A premature baby weighing one kilogram was born one night but deemed too small for the pediatric tents. No space was available at any of the other medical centres in the area, so we built a makeshift incubator out of a cardboard box, blankets and a desk lamp. Another newborn had clear-cut neonatal jaundice (though we could not get a measurement of how high the bilirubin level actually was). Until a bililight was found, the infant was placed on a cot in an internal courtyard in the direct sun, a throwback to how phototherapy for neonatal jaundice was first discovered at a London, England, Hospital more than 50 years ago. 
Many of the kids were malnourished in a way none of us had ever encountered. Many infants succumbed to dehydration or infectious diseases that would have been easily treated back home.

The tragedies were unrelenting through the last hour we were in Port au Prince. About 40 minutes before we left for the airport, a man brought in his five-hour-old daughter. She was cold, without palpable pulses and taking agonal breaths about once every $40 \mathrm{sec}-$ onds. We worked on her for over 20 minutes and were able to get her perfused and her heartbeat back to normal, but not to breathe on her own. We had to stop as there was nothing else we could do.
It was such a heartbreaking and pointless death: it turned out that the father had first taken the baby to another clinic, where he was told they could not help him because there were no pediatricians available. By the time he had hitched a ride on a motorcycle (with the baby in his arms) and come to us, it was too late.

What can be done to make things better?

Clearly, the earthquake was only the most recent in a series of devastating blows that Haiti has sustained over the years. It is very important to remember that the ongoing crisis only two hours away from Miami is not going to disappear anytime soon. There remains an urgent need for donations of money, supplies and equipment to organizations whose stated goal is to make Haiti functional and self-sufficient at a higher level than present, especially with respect to the medical system.

Medical personnel who can give of their experience and time are also very much in demand, as the need greatly exceeds availability. Moral support, too, is also very important, Haitians continually told us.

I was reminded of the words of Rabbi Tarfon, who lived in Judea in the first century: "It's not your job to finish the work, yet neither are you free to disregard it." - Dennis Rosen MD, Boston, Massachusetts

DOI:10.1503/cmaj.109-3272 